\title{
CORRECTION
}

View Article Online

View Journal I View Issue

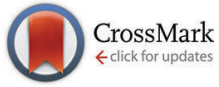

Cite this: J. Mater. Chem. B, 2017, 5, 1117

DOI: 10.1039/c7tb90011b

rsc.li/materials-b

\section{Correction: Advances in non-enzymatic glucose sensors based on metal oxides}

\author{
Hua Zhu, ${ }^{\text {ab }}$ Li Li, $^{\text {d }}$ Wei Zhou, ${ }^{\text {e }}$ Zongping Shao*de and Xiangjian Chen*ac \\ Correction for 'Advances in non-enzymatic glucose sensors based on metal oxides' by Hua Zhu et al., \\ J. Mater. Chem. B, 2016, 4, 7333-7349.
}

The name of one of the authors, Xiangjian Chen, was unfortunately spelled incorrectly as Xianjian Chen in the published article. The corrected author list is as shown here.

In addition, please note that in the Acknowledgements section, the grant number for Youth Project in Nature Science Foundation of Jiangsu Province should read Grant No. 20161006, and not No. 55126006.

The Royal Society of Chemistry apologises for these errors and any consequent inconvenience to authors and readers.

\footnotetext{
${ }^{a}$ Laboratory for Advanced Interdisciplinary Research, Center for Personalized Medicine/Institutes of Translational Medicine,

The First Affiliated Hospital of Wenzhou Medical University, Wenzhou, China. E-mail: wz1370@126.com

${ }^{b}$ Department of Obstetrics and Gynecology, The First Affiliated Hospital of Wenzhou Medical University, Wenzhou, Zhejiang, P. R. China

${ }^{c}$ Department of General Surgery, The First Affiliated Hospital of Wenzhou Medical University, Wenzhou, Zhejiang, P. R. China

${ }^{d}$ Faculty of Energy Science and Engineering, Nanjing Tech University, No. 30 South Puzhu Road, Nanjing, 211800, P. R. China. E-mail: shaozp@njtech.edu.cn

${ }^{e}$ Jiangsu National Synergetic Innovation Center for Advanced Materials (SICAM), State Key Laboratory of Materials-Oriented Chemical Engineering,

College of Chemical Engineering, Nanjing Tech University, No. 5 Xin Mofan Road, Nanjing 210009, P. R. China
} 